\title{
On the Spraying Modality of Liquids by Pyroelectrohydrodynamics
}

\author{
Sara Coppola, ${ }^{*}{ }^{\dagger}$ Giuseppe Nasti, ${ }^{\dagger}$ Veronica Vespini, ${ }^{\dagger}$ Valentina Marchesano, ${ }^{\ddagger}, \S$ \\ and Pietro Ferraro ${ }^{\dagger}$ \\ ${ }^{\dagger}$ Institute of Applied Sciences and Intelligent Systems E. Caianiello, CNR, Via Campi Flegrei 34, 80078 Pozzuoli, Italy \\ ${ }^{\ddagger}$ Centre for Integrative Biology-Univerity of Trento, via Sommarive n. 9, Povo, Trento 38123, Italy \\ ${ }^{\S}$ Trento Institute for Fundamental Physics and Applications, Università di Trento, Via Sommarive, 14, Povo, 38123 Trento, Italy
}

\section{Supporting Information}

ABSTRACT: We present for the first time an investigation on the spraying modality for the pyroelectrohydrodynamic (pyro-EHD) system. We show that the pyro-EHD spray (p-Spray) works well in the range from far field $(d>10 \mathrm{~mm})$ to near field $(d<2 \mathrm{~mm})$ without the need of external voltage and without the needs to change the experimental apparatus in that wide range of distances. Because the proposed method works without a counter electrode, no limitations are connected with the geometric shape of the substrate on which the spray coating is deposited. We report on several examples of the formation of disperse sprayed droplets, whose size can be varied between hundreds of micrometers and hundreds of nanometers, as well as on the production of sprayed pattern footpaths. The results reported here demonstrate the direct writing of spray patterns of nano/microdroplets with different materials in a very wide range, that is, from low- to high-viscous liquid solutions. Finally, as an example of application, we show the use of the p-Spray for the fabrication of silver-coated devices showing antimicrobial properties of the pattern produced by the p-Spray through a customizable and environmental friendly approach.

\section{INTRODUCTION}

Inkjet printing has attracted great attention for the fabrication of micro/nanofunctional devices as it is a low-cost technology that is easy to process and shows good material compatibility. Additionally, inkjet printing works in a noncontact mode; that is, it does not require a long-lasting and multistep process allowing complex functional patterns to be printed precisely on a flat and three-dimensional substrate with the help of moving platforms. In particular, the convergence of exceptionally superior output characteristics, low processing temperature, and solution processability makes possible the realization of a high-throughput and efficient fabrication process, thus ending the gap between academic prototypes and industrial standards. The industrial exploitation of the concept and use of inkjet printing are still growing up including different fields of applications in research, such as electronic and biomedical industries. Recently, increasing attention has been paid to electrospray as a suitable technique derived from the inkjet printing apparatus and used to obtain completely printed devices on large areas for different purposes and applications. Electrospray is an electrohydrodynamic (EHD) technology commonly used for film production. EHD spraying uses an intense electrical forcer to atomize liquids, generating charged droplets whose size can vary from micro- to nanoscale with a narrow size distribution. In classical EHD spraying, the liquid flows out of a capillary nozzle, which is maintained at a high electric potential. During the flow, the electrical charges are extracted from the fluid, and the internal electrical repulsion forces the liquid to form very small droplets. ${ }^{1}$ The electrostatic field can cause the liquid jet to be broken into smaller particles, as a consequence of the Columbian interaction of charges generated onto the liquid drop subjected to an electric field. ${ }^{2,3}$ There are many different methods used to fabricate printed films, including ultrasonic spraying, ${ }^{4}$ thermal evaporation, ${ }^{5}$ chemical vapor deposition, ${ }^{6}$ mechanical atomizers and molecule-by-molecule deposition onto a substrate, epitaxial plating, intercalation or implantation, and aerosol jet deposition. $^{7}$ The EHD spray system has several advantages over the aforementioned technologies. In fact, it represents a simple and cost-effective way to fabricate microthin films, in which droplets produced from a solution or suspension of selected materials are deposited directly to a target substrate. The advantages of spray by EHD include simple operational steps, an efficient process, an evenly deposited layer, and a good compatibility of materials. ${ }^{8,9}$ The electrospray techniques have been used for preparing numerous different thin films from biomaterials, ${ }^{10}$ to composite metals, ${ }^{11}$ ceramics, ${ }^{3}$ and nonmetal oxides. ${ }^{12-16}$ Conventional electrospray process uses

Received: June 20, 2018

Accepted: September 18, 2018

Published: December 19, 2018 


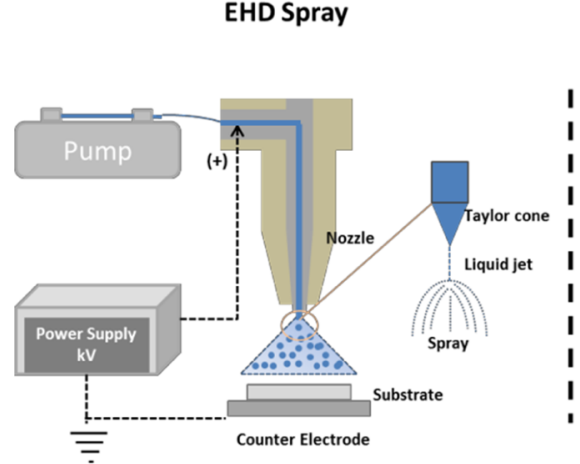

(a)
p-EHDS by surface oscillation

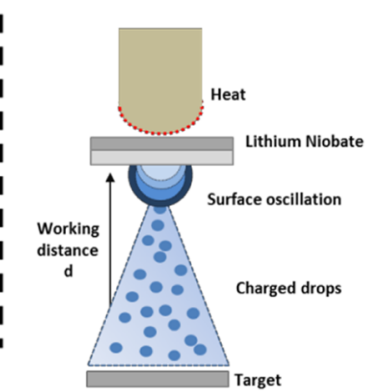

(b)

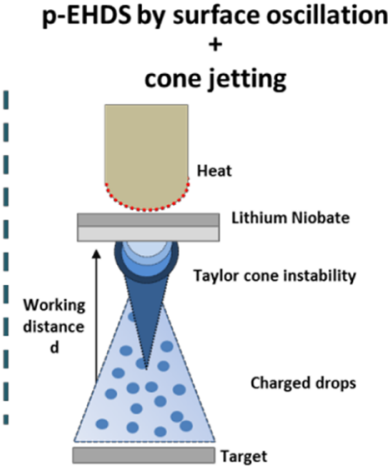

(c)

Figure 1. (a) Conventional EHD spray apparatus consisting of external power supply (kV), electrodes, liquid pump, and microengineered nozzle; the liquid is pumped through the nozzle forming the Taylor's cone form which the spray starts. The substrate must be included between the nozzle and the counter electrode. In the case of p-Spray, the activation is obtained by a temperature variation applied to the $\mathrm{LN}$ crystal, and the spray starts directly from the free surface of a liquid reservoir drop lying in front of the target of interest. Two possible evolutions regard the oscillation of the liquid surface that breaks into tiny droplets (b) and simultaneous cone elongation and droplet oscillation (c). The absence of counter electrode allows to print on several devices ready to use.

a working distance of $10 \mathrm{~cm}$ (far field) so that the deposition area is too large to collect the micropatterns. ${ }^{17,18}$ In order to minimize the deposition area and focus the printing cone, one effective way is to shorten this critical distance. The short distance of about $2 \mathrm{~mm}$ used in so-named "near-field electrospray" allows working where the jet is not fully opened up and a small sprayed footprint is obtained. ${ }^{19}$ Unfortunately, it has been demonstrated that at a short distance, a sharp tip needle may induce intense electrostatic stresses on the liquid meniscus because of its small radius ${ }^{20}$ and has to be replaced by a probe. The inconvenience of the set up modification is added to the complication of spray patterns by probe in the case of liquid solution and in particular for high-viscous polymer inks. ${ }^{19}$ In fact, the resulting electrosprayed patterns are not uniform; on the contrary, they appear different when produced under sufficient liquid supply at the initial period of the electrospray process, at the middle period when the solution inevitably shrinks, and at the final period when the solution covering the probe is insufficient. Moreover, this required a change of configuration, in terms of distance and of delivering ink, which would be a drawback for mass production technology. Actually, great interest has also been focused on the production of polymeric fibers films. Electrospinning (ES) has been deeply investigated as a technology for fiber production. ${ }^{21}$ In an ES polymer, solutions are ejected from the nozzle of a needle under a slight pressure while a voltage between the needle and the collector substrate leads to the formation and jetting of polymer fibers. ${ }^{22}$ Very recently, a novel method based on pressurized gyration (PG) has been proposed for processing polymer solutions for the mass production of polymeric fiber films, with high production rates that are orders of magnitude higher than classical ES. Unlike ES, PG does not require the use of an electric field, and it could be used for the fabrication of customizable film products with varying morphologies and properties. ${ }^{23}$ In this paper, we investigate and show for the first time a simpler process for achieving a liquid and polymeric spray based on the pyroelectric effect. It is very versatile, and it can be applied for many liquids and polymers without incurring in clogging effects. The pyroelectric effect is activated onto a lithium niobate (LN) crystal. ${ }^{24-27}$ We show that the pyro-EHD spray (p-Spray) works properly in a very wide interval of working distances, that is, in the range $2 \mathrm{~mm}<d<10 \mathrm{~mm}$. Noteworthy, the p-Spray is activated directly from the free surface of a liquid drop. As the p-Spray properties work at long distances, we prove here a controllable process for micro/ nanoscale fabrication, including on-demand traces and layerby-layer sprayed film deposition onto any target substrates, whereas in case of a short distance of $2 \mathrm{~cm}$, we demonstrate the formation of a sprayed pattern by the p-Spray, using a gap longer than the typical one adopted in the near-field application and thereby enlarging the opportunity of employment for spray coating. Moreover, by using the proposed configuration, the limit for the target thickness is completely removed ${ }^{28,29}$ because in the p-Spray proposed here, there is no counter electrode. In fact, we work in the forward mode, ${ }^{28}$ thus allowing ink printability directly on several devices of interest. In this work, by controlling the reservoir volume and keeping it constant during the spray time, we demonstrate a good control in the direct writing of disperse micropatterns of nanodroplets without using nozzles, probes, or templates. We have characterized the morphology of the pattern obtained by spraying different materials from low- to high-viscous liquids, using different liquids and polymer solutions. Compared to the traditional electrospray, the p-Spray allows a better control of the deposition, improving the functionalization and the performance of any device for different fields of applications. We propose the use of $\mathrm{p}$-Spray also for the fabrication of silvercoated devices showing antimicrobial properties, making easier the deposition step actually realized during the device assembling, through a customizable and environmental friendly approach.

\section{RESULTS AND DISCUSSION}

p-Spray Experimental Setup. In the more general case of EHD printing, the liquid flows through a capillary nozzle forming a meniscus at the end of the nozzle, and when the electric force overcomes the surface tension, a fine jet is ejected from the meniscus that finally breaks into a cloud of microand nanodroplets within the interaction of Coulomb's repulsion. Typically, in electrospray, there are two groups of forces that cause deformation and disruption of the liquid jet, that is, bulk forces on the jet, and normal and tangential stresses at the liquid surface. ${ }^{30}$ In the literature, different 
methods of producing sprays are reported, which differ by each other depending on the shape of the meniscus, the pattern of motion of the jet, and the mechanism of the formation of the droplets. Usually, a standard EHD apparatus for a far-field condition includes a nozzle and a counter electrode where droplets are formed by applying a high voltage using an external generator. This setup is usually expensive because it requires micro/nanofabricated nozzles, controlled flow liquid pump, and high-voltage power supply $(\mathrm{kV})$. The liquid is pumped and delivered through the nozzle, forming a Taylor cone from which the liquid jetting starts. The organization of the charged liquid droplets under the action of the EHD forces breaks the liquid emission into an aerosol jet attracted in the direction of the counter electrode (Figure 1a). In the case of pyroelectric effect, the jetting starts directly from the free surface of a liquid drop. When the liquid drop is subjected to the pyroelectric field, it forms an inverted triangle shape called "Taylor cone". At the end of the Taylor cone, the jet of liquid breaks up into a cloud of highly charged droplets because of the EHD forces. ${ }^{24,25}$ A complete cycle of EHD spraying usually occurs in a very short time and consists in meniscus deformation, oscillation-pulsation, and drop and/or jet separation from the capillary.

In the p-Spray, the pyroelectric effect induced by a temperature variation applied to the $\mathrm{LN}$ crystal drives the EHD pressure on the droplet reservoir. ${ }^{31,32}$ Figure $1 \mathrm{~b}$ shows the experimental setup, where an LN crystal is connected to a resistive probe used for the activation of temperature variation. A liquid drop is deposited on a pillar base previously realized on a standard glass coverslip to improve the uniformity of the base drops and positioned on the same side of the crystal. ${ }^{28}$ The $\mathrm{p}$-Spray works at ambient temperature (Tamb) preserving the great advantage of classical EHD, and a sudden temperature variation of about $30-40{ }^{\circ} \mathrm{C}$ is needed to be applied to the upper face of the LN crystal for the activation of the spraying cone. Nevertheless, the temperature experienced by the liquid droplet is about $10{ }^{\circ} \mathrm{C}$ lower than the value measured on the crystal; in the case of some biological molecules, this temperature should be below the value critical for cell damage and death. However, in a previous work, we have already demonstrated in the classical pyroprinting configuration that the heating due to the temperature variation did not cause damage on biological molecules for such rapid temperature variations. ${ }^{33}$ Still, it is important to note that the p-Spray can be activated not only by increasing the temperature but also equally well by lowering it. In fact, the pyroelectric effect, if induced by the absolute temperature gradient, changes independently by the sign of temperature variation. Therefore, in specific cases in which biomolecules or cells can suffer damage, it is possible to adopt the strategy to lower the temperature instead of increasing it.

When the electric fields exceed a critical intensity, the droplet starts to deform under the action of the sufficiently strong pyro-EHD field and releases from the conical tip structure a simultaneous emission of microdroplets monodispersed and sprayed with high accuracy onto the target. Two typical meniscus profiles of the liquid drop are possible as a function of the material: (1) the oscillation of the liquid surface that breaks into tiny droplets (see Figure 1b) and (2) the simultaneous cone elongation and droplet oscillation ${ }^{34,35}$ as illustrated in Figure 1c.

These pulsations continue until the fields dissipate on cooling of the pyroelectric material, and the ejection of the droplets can therefore be reversibly turned on and off in this manner. The heating creates a sort of "virtual nozzle" through localized EHD effects, thereby omitting the need for real nozzles. In fact, the proposed spraying process is triggered by a temperature variation ${ }^{24,25}$ introducing an important simplification of the conventional inkjet printing apparatus.

During the experiment, the p-Spray effect was recorded using a complementary metal-oxide semiconductor camera integrated in a visualization line made of a light-emitting diode blue source and a $5 \times$ objective. The spray process is very fast and lasts only few microseconds. It cannot be detected by human eye, but information about the spraying process can be retrieved analyzing frame-by-frame the recorded movie. A typical image frame is reported in Figure 2; the atomized

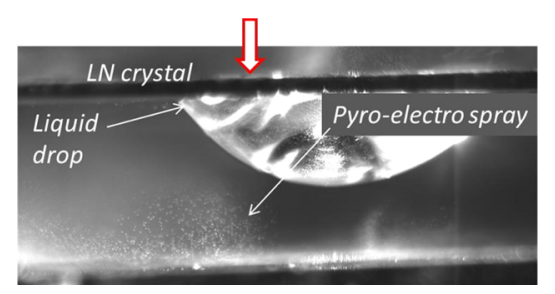

Figure 2. Single frame extracted from the experimental movie showing the direction of heating, the profile of the liquid drop, and the cloud of pyro-EHD-sprayed droplets.

droplets are barely visible around the liquid drop. The droplets are ejected from the reservoir as a consequence of the activation of the pyroelectric effect. The sprayed droplets are collected onto the target substrate. In our experiment, a commercial microscope slide glass was used as the target substrate just for the characterization of the spray process. It is important to point out that the forward configuration adopted for the pyro-EHD Spray will allow direct printing on every kind of substrate of several devices ready to use, enlarging the application of spray deposition of conventional EHD systems, where the thickness of the substrate/device represents a geometrical constrain and as a consequence a severe limitation. ${ }^{28,29}$

The results we report here show how the pyrospraying is obtained by appropriate selection and control of solution, polymer properties, and EHD forces. ${ }^{36-38}$ This could be seen as an intrinsic limitation of our method, but we believe that this article could be a first insight into the novel p-Spray approach, furnishing some guidelines to experiment and comprehend it. Further studies on the modeling, simulation, and experimental works will be necessary in order to understand the process physics, predict the process outcome, and guide the process control. However, in order to investigate these problems, we started with the characterization of different solutions in terms of viscosity and surface tension using the p-Spray (Table 1). As a first result of our work, we observed that the solutions characterized by the mean value of surface tension and low viscosity represent the optimum condition of monodisperse p-Spray. In general, as reported in Table 1, the pyro-EHD approach could be applied to different liquids (inks) having various properties. Among the various experimental parameters, the surface tension and the viscosity of the fluid deeply affect the spraying, spinning, and printing process. In particular, the surface tension opposes the electric field applied at the fluid interface and affects the ability of a 
Table 1. Boiling Point $\left({ }^{\circ} \mathrm{C}\right)$, Viscosity (cP) Measured at 25 ${ }^{\circ} \mathrm{C}$, and Surface Tension $(\mathrm{mN} / \mathrm{m})$ of the Inks Experimented Using Pyro-EHD

$\begin{array}{llcc}\text { material } & \text { Teb }\left({ }^{\circ} \mathrm{C}\right) & \text { viscosity }(\mathrm{cP})\left(25^{\circ} \mathrm{C}\right) & \text { surface tension }(\mathrm{mN} / \mathrm{m}) \\ \text { NMP } & 202 & 1.655 & 40.8 \\ \text { DMF } & 153 & 0.802 & 37.1 \\ \text { GLY } & 290 & 1.41 \times 10^{3} & 64.0 \\ \text { TTD } & 254 & 2.13 & 26.6 \\ \text { mOIL } & 800-870 & 2.42 \times 10^{3} & 450 \\ \text { PDMS } & & 3.50 \times 10^{3} & 19.9\end{array}$

liquid to realize electrospray while the viscosity opposes the fluid to atomize.

According to that, we experimentally observed that a high surface tension increases the threshold electric field for an ejection, whereas a high viscosity increases the size of the deposited droplets. For this reason, solutions with a high viscosity and low surface tension [i.e. plasma desorption mass spectrometry (PDMS)] could be pyropatterned with a high resolution in the forward configuration, ${ }^{25,28}$ whereas highviscous inks (i.e. mOIL) with a high surface tension are the best candidates for pyroprinting in the conventional configuration achieving printing of pico-nanodroplets. ${ }^{39,40}$ The solutions [i.e. inks 1-methyl-2-pyrrolidinone (NMP), N,Ndimethylformamide (DMF), and glycerol (GLY)] characterized by the mean value of surface tension and low viscosity represent the optimum condition of monodisperse droplets by p-Spray (as demonstrated in the following) while p-Spray spinning would be possible working with low viscosity and low surface tension solution (Ag NMP ink and AgTTD ink). Focusing on the spray approach, here we present three different typologies of $\mathrm{p}$-Spray in the function of the nature of the ink. It is important to note that by p-Spray, the jetting could be switched to spraying patterning modality without the need of probes, nozzles, and stamps. First, we report on a monodisperse p-Spray using three different liquids (i.e. NMP, GLY, and DMF) and thus showing the formation of a uniform deposition of small drops in the size range from hundreds of micrometers to hundreds of nanometers. The characterization of the prepared samples was done using an optical microscope. Deposition and distribution of tiny droplets onto the target substrate were observed and measured. We also studied the effect on the deposition quality changing the distance between the reservoir and the target substrate. Moreover, we used a polymeric solution of polyvinylpyrrolidinone (PVP) in ethylene glycol (EG) noticing a very different behavior where a continuous liquid jet is spray-spun as a structured pattern of drops. In case the of the polymer solution, a more complex pattern can be printed. In fact, the sprayed droplets are also affected by the whipping instability, thus tracing the typical mats usually achieved by ES. Finally, as an example of application, we tested spraying of Ag inks (NMP and TTD) realizing patterns that could be useful for controlling antibacterial properties of functionalized surfaces. On the theoretical side, the multiphysical mechanism of electrospray has not been understood in full. Instability analysis involves multiple simplifications and assumptions and may not reflect the actual process conditions, although the analysis results are qualitatively coincident with the experimental results. We believe that an additional theoretical and experimental investigation could improve the comprehension of the $\mathrm{p}$ Spray approach for different inks.

Organic Solvent Spray. We made analysis and characterization of the p-Spray process in order to have a better control on printing. Starting from a drop reservoir of $500 \mu \mathrm{L}$, we applied the pyroelectric effect and observed the resulted printing at well-defined working distances $d$ between the liquid reservoir and the target substrate: $2,4,7$, and $12 \mathrm{~mm}$.

Monodisperse sprayed droplets over a large area could be realized using the solutions with medium values of viscosity and surface tension. In particular, the solutions NMP, GLY, and DMF in Table 1 were pyrosprayed and the pattern realized by pulsation/oscillation of the droplet. The working distance used for the spray activation reported is $4 \mathrm{~mm}$. The measured distribution is quite regular and comparable in terms of dimension and distribution to conventional spray techniques (Figure 3). The diameters of the droplets produced are estimated to fall in the range $0.5-10 \mu \mathrm{m}$. The results have been validated for the NMP ink even in the case of a uniform dispersion of silver nanoparticles (NPs) (see Materials and Methods). Controlling the working distance, it is possible to vary the spot dimension of the spray produced and the distance between the adjacent sprayed droplets. The spray in the case of solvents characterized by a mean value of surface tension and viscosity (NMP, DMF, and GLY) assumes a circular shape centered in the region of the starting drop and made up monodispersed droplets. The bigger central drops are produced by the simultaneous elongation of the Taylor's cone with the drop oscillation. Figure 4 shows the sprayed patterns obtained at two different distances 4 and $12 \mathrm{~mm}$, respectively, and by three consecutive sprayed shots. By increasing the working distance, the spotted area became wider, and in the case of multiple sprays, the droplets localized in the region laying in front of the ink reservoir collapsed with each other assuming volumes and dimensions different from the ones in the peripheral regions.

In our experiments, we observed that in the case of little sprayed droplets (radii $1-2 \mu \mathrm{m}$ ) of volatile solvents, the realized pattern could be difficult to detect. In fact, for DMF and NMP, the evaporation of the solvent is very fast, and the available time for the spray observation is limited. Thus, an

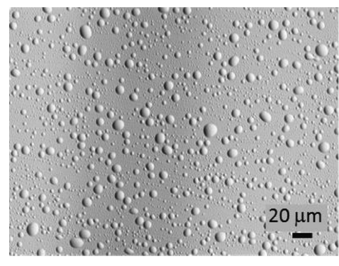

(a)

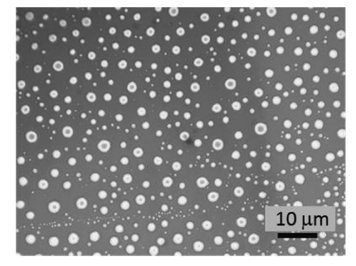

(b)

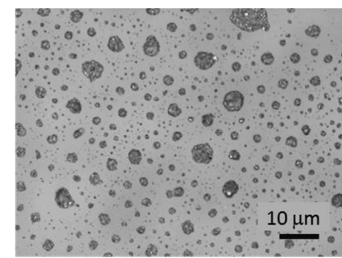

(c)

Figure 3. Monodisperse sprayed droplets of DMF (a), GLY (b), and NMP (c). The distribution is obtained by a single pyro-EHD shot at a working distance of $4 \mathrm{~mm}$. The target is a commercial glass coverslip. 


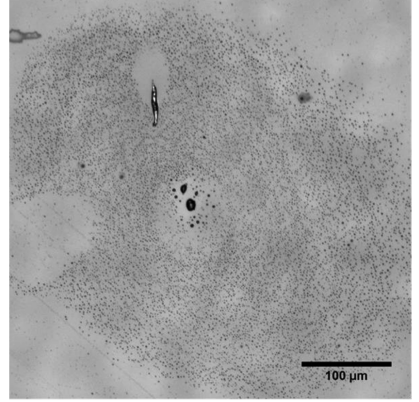

(a)

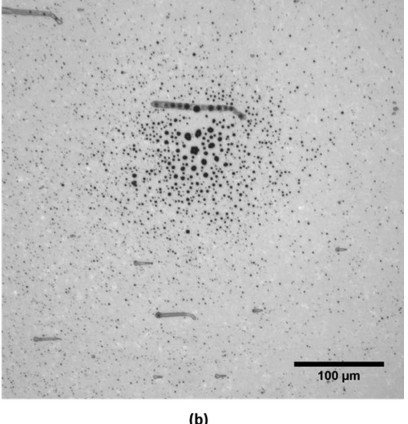

(b)
Figure 4. Sprayed patterns at two different working distances 12 (a) and $4 \mathrm{~mm}$ (b) obtained by three consecutive shots. When the target moves closer to the reservoir drop, the sprayed area becomes smaller.

alternative way for visualizing the printed pattern for enhancing the comprehension of the spray printing approach was adopted. In this case, a yellow fluorescent (Lumogen $\mathrm{F}$ yellow 083, by BASF) dye was added with a concentration of $10 \mathrm{wt} \%$ to their base solutions. On the contrary, GLY ink presented much lower vapor pressure compared to inks DMF and NMP; this allowed the use of ink GLY as it is without the addition of a fluorescent dye. In the case of inks DMF and NMP after the spraying and once the solvent was evaporated, the fluorescent signal of the dyes was observed on a fluorescence microscope. In this way, it was possible to visualize the sprayed area for identifying the positions of the dispensed drops, thus allowing the full characterization and analysis of the printed patterns. The measured values are reported in Figure 5.

As expected, the extension of the sprayed area depends on the working distance between the drop and the target. Figure $6 \mathrm{a}$ reports the plot describing this correlation between the target-to-drop distance and the radius of the sprayed area. The observed trend holds for all the different solutions tested, and the radius increases from $0.5 \mathrm{~mm}$, when $d=2 \mathrm{~mm}$, to the maximum of about $4 \mathrm{~mm}$, reported in the case of DMF solutions, when $d=12 \mathrm{~mm}$. Increasing the dimension of the sprayed drops leads to the increase of the distance between the adjacent droplets (Figure 6b). This behavior is easily understood under the assumption that the volume of the material ejected for each test is constant and that the droplets form a conical shape during the flight time. Therefore, the key parameters for the control of the process and of the produced patterns are the type of ink, the time of deposition, and the distance between the reservoir and the target. The shape of the sprayed jet resembles a truncated cone. The parameters governing the area of the spray are therefore the diameter of the starting drop $(r)$, the distance between the drop and the target $(d)$, and the angle of inclination of the cone $(\alpha)$. Because of the geometric consideration, the final area of coverage of the spray is $A=\pi \times(r+d \sin (\alpha))^{2}$. In the previous equation, the $r$ and $d$ parameters are purely related to the working condition and can be adjusted as needed (in a limited range). The $\alpha$ parameter is lightly related to the electric field generated by the pyroelectric crystal and strongly related to the nature of the fluid. For this reason, it is not easy to exactly predict the final area of the spray, but it should be experimentally determined whenever a new solvent is used.

Jet Instability Spray of Polymeric Solutions. As previously described, the electrospray takes place during the inkjet printing process by controlling the experimental parameters. However, the spray modality it would be feasible a)

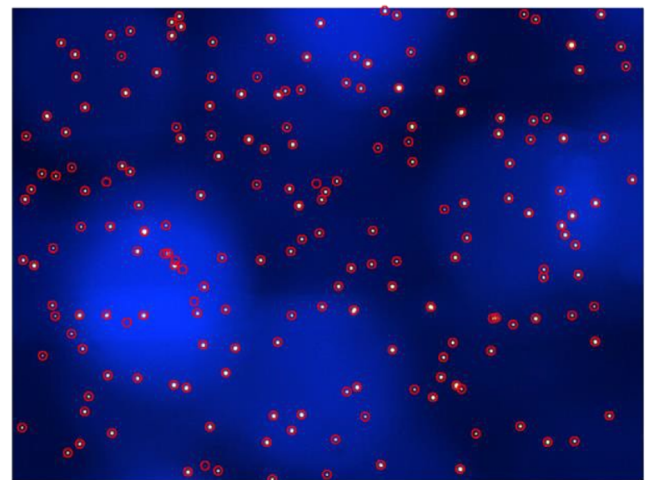

b)

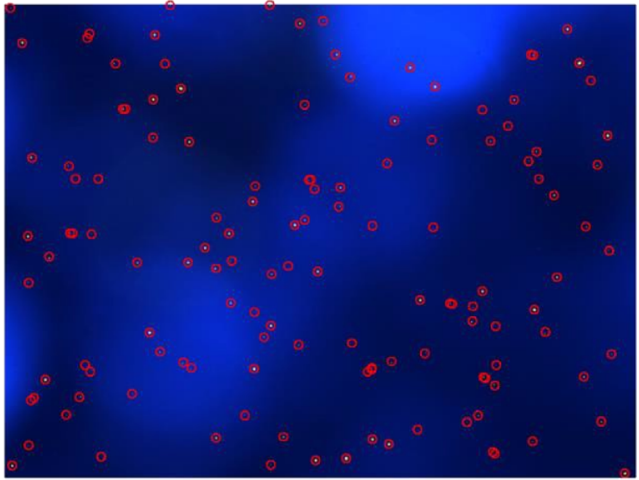

c)

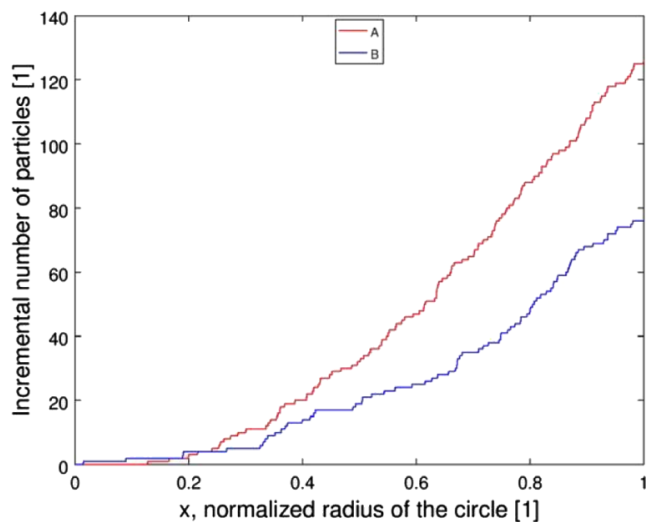

Figure 5. Fluorescent image acquired using the ink NMP doped with yellow fluorescent dyes. The shadow of the sprayed droplets is not visible, but their position is represented by the dyes. (a) Image obtained by pyro-EHD-Spray at a working distance of $7 \mathrm{~mm}$. (b) Image obtained by pyro-EHD-Spray at a working distance of $12 \mathrm{~mm}$; in this case, the sprayed droplets are more distant from each other. (c) Incremental number of fluorescent particles included into circles of normalized radius $x$. The red line refers to (A) and the blue line refers to (B).

even during the ES in the case of diluted polymer solutions. Usually, in ES, polymer solutions are ejected from the nozzle of a needle under a slight pressure while a voltage between the needle and collector substrate leads to the formation of a Taylor's cone and starts jetting. Under appropriate conditions, this jet forms a thin and continuous filament and is deposited on a target substrate with true microscale regularity. ${ }^{31}$ More recent methods avoid the electric filed and use solution blowing and centrifugal spinning to produce large quantities of homogenous fibers with a great control over the final product morphology because of the increased number of parameters that can be finely tuned. ${ }^{23,41}$ The forward configuration here proposed for the spray technique works even in the case of ES 


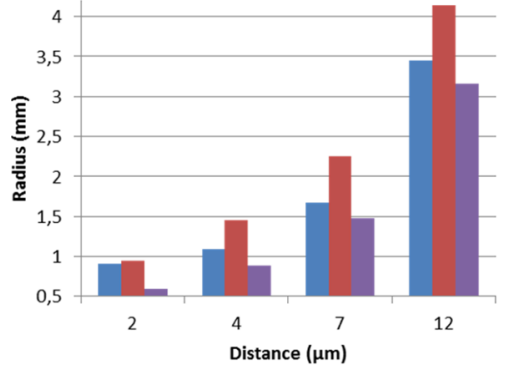

(a)

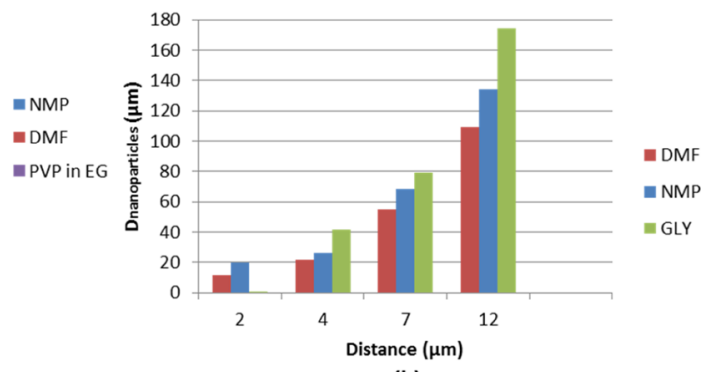

(b)

Figure 6. Characterization of the pyro-EHD-Spray obtained by moving the working distance from 2 to $12 \mathrm{~mm}$. The solutions used for the characterization are NMP, GLY, and DMF; the distances used in the evaluation are 2, 4, and 7 and $12 \mathrm{~mm}$. (a) By increasing the target-to-drop distance, the radius of the sprayed area consequently increases with a parabolic trend. (b) By increasing the dimension of the sprayed drop, the distance between the adjacent droplets subsequently increases.

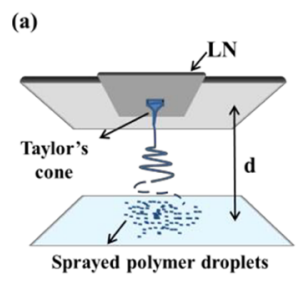

(b)

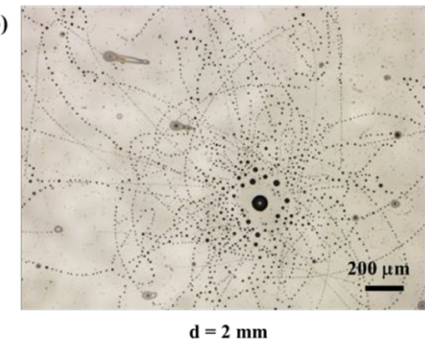

(c)

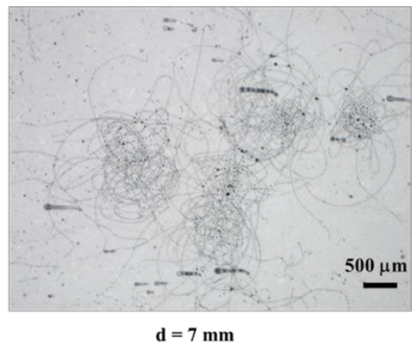

Figure 7. (a) Outline of the pyro-EHD-Spray; in the case of polymer solution, the liquid jet is subjected to bending and whipping instabilities very common in conventional ES. Sprayed polymer patterns made of spiral drops when $d=2 \mathrm{~mm}(\mathrm{~b})$ and $d=7 \mathrm{~mm}(\mathrm{c})$ are intercepted onto the target.

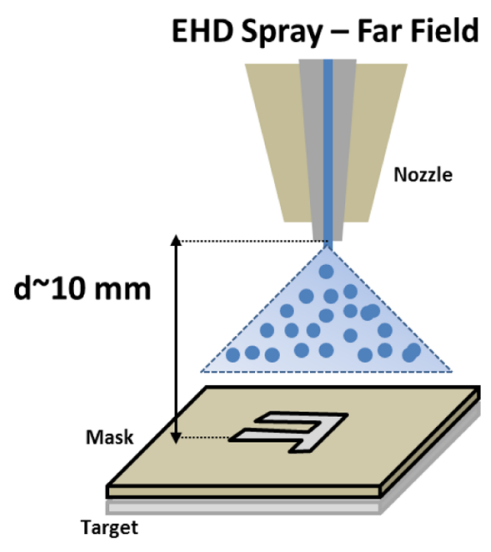

(a)

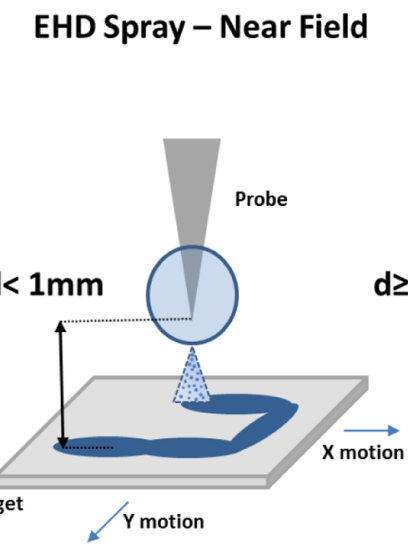

(b)
PYRO Spray

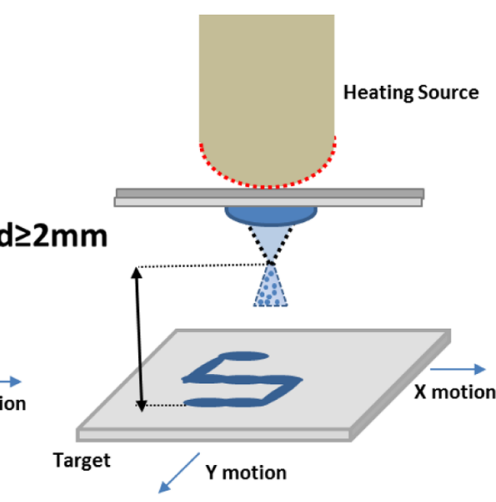

(c)

Figure 8. In the case of spray patterning, the conventional EHD setup must be modified. (a) In the case of a working distance of $10 \mathrm{~mm}$ (far field), a template must be placed over the target reporting the profile required for patterning. In this case, every kind of experiment focused on a welldefined pattern that needs a specific and designed mask, protracting the time required for fabrication and becoming more expensive. (b) In order to use the conventional EHD system but to avoid the use of masks, the working distance must be reduced to about $1 \mathrm{~mm}$. In this case, the intense electrostatic stress grows on the liquid profile, and the nozzle must be replaced by a tip to allow a stable ejection process that could be used for spray patterning. (c) In the case of the setup based on the pyro-EHD effect, the distance must be shortened to about $2 \mathrm{~mm}$, and the jetting cone will be already focused for patterning sprayed droplets with no invasive or complicated alteration of the apparatus.

using the polymer solution, such as PVP solution in EG (Figure 7a). These two forms of printing, spraying and spinning, are two aspects of the same technology. We observed that, keeping the temperature constant, the spinning results were obtained in the case of solution with the polymer amount over the $10 \%$ in fact, in the case of lower polymer content, we observed monodisperse spray formation, as shown in Figure 3. Keeping constant the activation temperature of the pyro-EHD effect, the increase of viscosity causes p-spraying to become $\mathrm{p}$ spinning. The PVP polymer was used here for demonstrating the reliability of the technique because of its widespread use and the wide range of available solvents and concentrations. Once applied the pyroelectric effect, we observed the formation of an unstable Taylor's cone, so that under the action of the pyroelectric field, the flying polymer fiber broken up into separated droplets. This is due to the disruption of the continuous jet into single drops activated by the electrical instability of the accumulated charges. The break-up formation depends in fact on the unbalance between the external electric charges and the surface charges on the polymer solution. In particular, once the electric charges overcome the surface ones, the thin polymer jet collapses into separated droplets. The jet 
began to oscillate in both the radial and axial directions, and the amplitude of oscillations increased further downstream. The jet became thinner before it broke up into droplets because of ink instabilities. ${ }^{42}$ According to the form of disintegration, more detailed spraying patterns such as jetting, whipping, and unsteady cone jet were classified. These droplets are influenced by the motion acquired during the bending instabilities and intercept the target substrate into spiral drops patterns. In the case of $2 \mathrm{~mm}$ distance, the spiral distribution appears as a random mat of separated droplets (Figure $7 \mathrm{~b}$ ), whereas increasing the spinning distance, the bending instabilities arise, and the distribution is consequently more chaotic (Figure 7c). The Taylor's cone is very unstable at a longer distance and moves spatially over the target substrate losing the focusing properties visible at a smaller distance. With the method proposed, sprayed and spun spiral structures are obtained by an easy and versatile approach based on a singlestep process, thus avoiding repetitive, time-consuming, and expensive lithographic procedures, or direct printing by mechanical scanning.

Silver Ink Patterned Spray. Using the proposed p-Spray approach and the working distance of $2 \mathrm{~mm}$ in the case of silver-based ink, the jetting cone could be focused for printing a restricted pattern made of adjacent sprayed droplets onto a small area of interest. In particular, for the first time, in the case of printing sprayed pattern, the working distance has not to be shorten below $1 \mathrm{~mm}$ as it happens in the case of near-field $\mathrm{ES}^{43,44}$ where the nozzle-to-substrate has to be controlled in order to minimize the deposition area. The pattern realized by spray droplets could be very interesting in terms of biological response; in fact, printing patterns with topography can be much more effective than simply depositing a continuous coating on the substrate. Usually, in classical spray for the functionalization of a line or also a small area, it must be used as a template intercepting the sprayed cloud so that after removing the template, we could have a negative stamp. Commonly various template/molding strategies are utilized to fabricate micro/nanopatterns in far-field condition, so that patterns are formed on the substrate after removing a special designed template, ${ }^{36,37}$ which covers the target (Figure $8 \mathrm{a}$ ). The setup is very similar to the one proposed for classical EHD spray, and the fabrication of the sprayed pattern is a direct result of the template adopted, so that additional time and template fabrication costs have to be added to the already expensive EHD apparatus. In order to simplify the setup, additional experiments at a shorter distance have been conducted to allow a stable ejection process. Near field refers to the regions where the jet is not fully opened up and a small foot print is expected (Figure 8b). Unfortunately, it has been demonstrated that sharp tip needles may induce intense electrostatic stresses on the liquid meniscus because of its small radius. In this case, one will have to change the nozzle with a probe that may be a limit in the case of printing a continuous liquid pattern in general and in particular high-viscous polymer ink. Here, we show how in the case of silver-based ink that the same setup used for the monodisperse spray approach could be easily adapted to spray patterning by only adjusting the working distance (Figure $8 \mathrm{c}$ ).

The p-Spray once activated onto the Ag inks in TTD and NMP, characterized by a very low value of viscosity and surface tension, could lead to the direct printing of sprayed patterns. This process can be used to deposit liquid suspension at different concentrations. The limitation is related to the viscosity and to the surface energy of the system. Very concentrated suspensions, depending on the specific nature and interaction of the solvent and the dispersed particles, can lead to a change of behavior during the deposition, going from the spray of very small drops to the spinning of continuous jets. In the case of TTD ink, we used a concentrated suspension of metal ink at $\sim 50 \mathrm{wt} \%$; the spraying results are comparable with those of conventional EHD used to atomize the dense suspension of NPs with high solid concentrations. ${ }^{45}$ In our work, the advantages of the $\mathrm{p}$-Spray process over conventional electrospray is to control the deposition of droplets for spray pattern coating and for the fabrication of continuous and/or disconnected small area patterns without the need of nozzles, probes, molds, or masks. The final pattern design will be defined by just controlling the motion of the target substrate. For this experiment, the target substrate was mounted on a two-axis motor stage. For the movement of the stage, we used two stepper motors, consisting of a high-precision linear motor with an $x-y$ axis (SGSP26-100(XY) SIGMA KOKI CO., LTD) and a computer numerical control board (SIGMA KOKI CO., LTD). The software was generated by a MATLAB script and used to control the printing pattern and the moving speed along each axis during the spray process. The patterns designed for the experiments described in the following are very simple and consist in separated droplets or a continuous line realized by varying the moving speed, in order to study the focused spray cone. Figure 9a shows the deposited sprayed

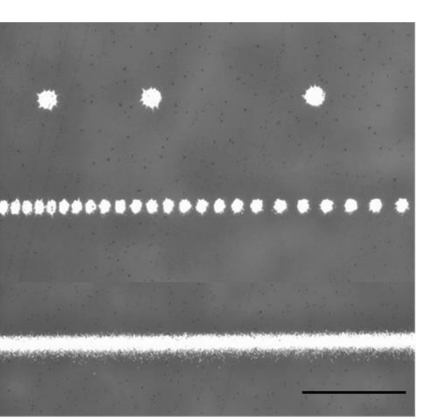

(a)

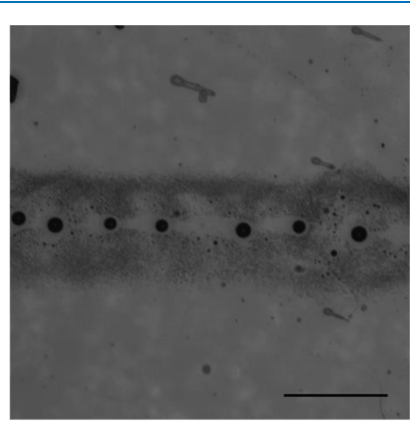

(b)
Figure 9. Spray patterning through the pyro-EHD effect in the case of two different silver-based inks: (a) sprayed patterns obtained using the Ag-NMP ink, examples of printing separate sprayed droplets with different periods (up and halfway) and a sprayed line (bottom). (b) Commercial Ag ink in TTD solvent; in this case, the spray is less focused, and we observe the formation of a central line of droplets surrounded by other little sprayed droplets. Scale bars $50 \mu \mathrm{m}$.

dots with different periods and dimensions and a comparison of deposited sprayed dots and line. In the first case, controlling the deposition speed, it is possible to switch from the deposition of separated droplets to a continuous line. In this case, the target speed is decreased to $0.2 \mathrm{~mm} / \mathrm{s}$ such that atomized particles overleap into larger droplets. In the case of silver ink based on NMP solvent, the sprayed pattern is quite regular and focused on restricted areas (Figure 9a), making possible the patterning of separated droplets and sprayed lines. The ink used for the experiment reported in Figure $9 b$ is instead a silver NP based commercial solution made up of TTD solvent (ULVAC GmbH); in this case, the profile appears less regular.

Antibacterial Spray. Biofilms are detrimental to human life and industrial processes because of potential infections, 
contaminations, and deterioration. The evaluation of microbial capability to form biofilms is of fundamental importance for assessing how different environmental factors may affect their vitality. ${ }^{46}$ Therefore, the development of new methods for controlling and reducing the bacterial growth are still investigated. ${ }^{47}$ Recent works demonstrate the fabrication of tellurium-loaded polymeric fibers and fiber meshes with antimicrobial properties tested especially against the Escherichia coli strain K12. ${ }^{48}$ Moreover, it is well-known that silver and silver-based compounds exhibit a strong antibacterial activity to a wide range of microorganisms which have been used extensively in many bactericidal applications. ${ }^{49}$ For this reason, Ag-loaded nylon nanofibers have been used for a wide range of biological applications, such as an antibacterial, wound dressing, functional scaffold for tissue engineering, and showing bacterial killing rates ${ }^{41,50}$ reaching $\sim 100 \%$. In this work, the antimicrobial properties of the p-Spray were proved in the case of silver-based spray using an NMP solution of $\mathrm{Ag}$ NPs (see Materials and Methods section). In particular, the NMP was chosen as a base ink and as a carrier for the formation of a monodisperse pattern of silver NPs. The pSpray was first used for the functionalization of a commercial Petri dish where bacteria usually grow. Once the silver solution was sprayed, the Petri dish was sterilized and subjected to temperature evaporation for $24 \mathrm{~h}$ in order to remove all the remaining solvent. After that, the bacteria (E. coli DH5-alpha) were incubated in the cell culture medium over the sprayed patterns for $24 \mathrm{~h}$ at $37{ }^{\circ} \mathrm{C}$. The antibacterial mechanism of $\mathrm{Ag}$ NPs has not yet been completely understood. The most possible mechanism is due to $\mathrm{Ag}^{+}$released from $\mathrm{Ag} \mathrm{NPs,} \mathrm{which}$ strongly bind to thiol groups found in enzymes and proteins on the cellular surface and can interfere with cell division and lead to bacterial cell death. ${ }^{51}$ Moreover, Ag NPs can cause oxidative damage with producing reactive oxygen species, leading to attack enzymes and proteins and resulting in irreversible damage to DNA replication. ${ }^{52}$ In our experiment, we observed that after $24 \mathrm{~h}$ of incubation, the arrangement of bacteria was different and the shape was changed from an elongated to a circular one, as reported in the images of Figure 10. This

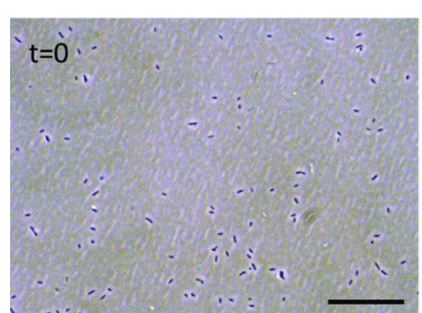

(a)

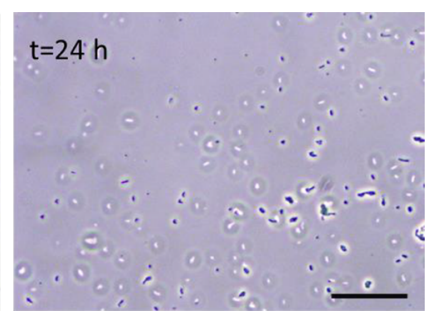

(b)
Figure 10. (a) Bacteria incubated in a cell culture medium over a microscope glass covered with a monodisperse spray of Ag-NMP ink at time $=0$. (b) After $24 \mathrm{~h}$ of incubation at $37^{\circ} \mathrm{C}$ in a shaker, the shape is changed from an elongated to a circular one, indicating the bactericide effect of the silver sprayed on the target through p-Spray. Scale bar $50 \mu \mathrm{m}$.

observation leads us to hypothesize that the bactericide effect of the silver NPs sprayed on the target through p-Spray affected the bacteria in terms of duplication and growth. The antibacterial behavior displayed a dose-dependent manner, ${ }^{53}$ so that by controlling spray time, we could control the dose dispensed to bacteria.

\section{CONCLUSIONS}

In summary, an environmentally friendly, facile, and simple method was demonstrated for the spray printing approach activated by pyroelectricity. We demonstrated that $\mathrm{p}$-Spray could be activated directly from the free surface of a liquid drop. Moreover, we show that the proposed method works well in the range from far field to near field without the need of external voltage and without the needs to change the experimental apparatus. Furthermore, we showed that the proposed method works without a counter electrode so that no geometrical limitations are connected with the shape of the target substrate enlarging the opportunity of employment for spray coating. We reported on several examples of the formation of disperse sprayed droplets, whose size can be varied between hundreds of micrometers and hundreds of nanometers, as well as on the production of sprayed pattern footpaths using various kinds of solvents and polymers. In the case of an E. coli bacterial culture, the application of silverbased spray coating showed a good antibacterial activity, demonstrating that $\mathrm{p}$-Spray could be a promising technique for application in a wide range of biomedical applications. Even if further development of the process would require both theoretical and experimental efforts, our results showed a promising technique that could be used as a bottom-up approach very useful in nanotechnology and for biomaterials where a nanostructure is usually built from elementary components.

\section{MATERIALS AND METHODS}

DMF $\left(\mathrm{C}_{3} \mathrm{H}_{7} \mathrm{NO}\right)$, NMP $\left(\mathrm{C}_{5} \mathrm{H}_{9} \mathrm{NO}\right)$, EG $\left(\mathrm{C}_{2} \mathrm{H}_{6} \mathrm{O}_{2}\right)$, and glycerol $\left(\mathrm{C}_{3} \mathrm{H}_{8} \mathrm{O}_{3}\right)$ were purchased from Sigma-Aldrich and used without further purification. Fluorescent dyes (Lumogen F yellow 083, by BASF) were added to DMF and NMP at a $10 \%$ concentration as a marker to improve the visualization and analysis of the spray. Two solutions of PVP $\left[\left(\mathrm{C}_{6} \mathrm{H}_{9} \mathrm{NO}\right)_{n}\right.$, $M_{\mathrm{w}}=360000$, bought from Sigma-Aldrich] in EG were prepared at a $35 \%$ concentration. PVP was added to EG for $1 \mathrm{~h}$ at $50{ }^{\circ} \mathrm{C}$ under magnetic stirring. In addition, a commercial silver ink (Ag Nanometal ink, purchased from ULVAC, dissolved into tetradecane (TTD), $\mathrm{C}_{14} \mathrm{H}_{3} \mathrm{O}, \mathrm{TTD}$ ) and a dispersion of silver nanopowder (Ag 99\%, Sigma-Aldrich, $<150$ $\mathrm{nm}$ ) in NMP (AgNMP) were tested in order to test its bactericide activity.

E. coli DH5-alpha was plated and incubated on agar plates. The day before the beginning of the experiment, a single bacterial colony was picked up and cultured in a Luria-Bertani (LB) broth medium $(10 \mathrm{~g} / \mathrm{L} \mathrm{NaCl}, 10 \mathrm{~g} / \mathrm{L}$ tryptone, $5 \mathrm{~g} / \mathrm{L}$ yeast extract) at $37{ }^{\circ} \mathrm{C}$ in a shaker incubator for $16-18 \mathrm{~h}$ to achieve saturation conditions. A 1:5 volumetric dilution of cell culture was then grown in the LB medium until reaching the log phase corresponding to a cell concentration of $4 \times 10^{8}$ cells $/ \mathrm{ml}$, verified by OD measurements at $600 \mathrm{~nm}$. The cells were then centrifuged at $5000 \mathrm{rpm}$ for $10 \mathrm{~min}$ in order to separate the cells from the medium and then resuspended in a fresh $\mathrm{LB}$ medium to reach a concentration of $2 \times 10^{7}$ cells $/ \mathrm{mL}$.

\section{ASSOCIATED CONTENT}

\section{Supporting Information}

The Supporting Information is available free of charge on the ACS Publications website at DOI: 10.1021/acsomega.8b01398.

Activation of p-Spray on a drop of glycerol (AVI) 


\section{AUTHOR INFORMATION}

\section{Corresponding Author}

*E-mail: sara.coppola@cnr.it.

ORCID

Sara Coppola: 0000-0002-2795-0079

Giuseppe Nasti: 0000-0003-0867-2402

Veronica Vespini: 0000-0001-5793-6860

Pietro Ferraro: 0000-0002-0158-3856

\section{Notes}

The authors declare no competing financial interest.

\section{ACKNOWLEDGMENTS}

The authors thank Federico Olivieri and Michele Todino for technical assistance in the acquisition of $\mathrm{p}$-Spray images.

\section{REFERENCES}

(1) Blades, A. T.; Ikonomou, M. G.; Kebarle, P. Mechanism of electrospray mass spectrometry. Electrospray as an electrolysis cell. Anal. Chem. 1991, 63, 2109-2114.

(2) Li, X.; Huang, J.; Edirisinghe, M. J. Novel patterning of nanobioceramics. Template- assisted electrohydrodynamic atomization spraying. J. R. Soc., Interface 2008, 5, 253-257.

(3) Duan, H.; Li, C.; Yang, W.; Lojewski, B.; An, L.; Deng, W. Nearfield electrospray microprinting of polymer-derived ceramics. $J$. Microelectromech. Syst. 2013, 22, 1-3.

(4) Jayaraman, V. K.; Álvarez, A. M.; de la Luz Olvera Amador, M. A simple and cost-effective zinc oxide thin film sensor for propane gas detection. Mater. Lett. 2015, 157, 169-171.

(5) Nguyen, T.; Tuan, N. T.; Nguyen, V. D.; Cuong, N. D.; Kien, N. D. T.; Huy, P. T.; Nguyen, V. H.; Nguyen, D. H. Near-infrared emission from $\mathrm{ZnO}$ nanorods grown by thermal evaporation. $J$. Lumin. 2014, 156, 199-204.

(6) Huang, C.-C.; Al-Saab, F.; Wang, Y.; Ou, J.-Y.; Walker, J. C.; Wang, S.; Gholipour, B.; Simpson, R. E.; Hewak, D. W. Scalable highmobility MoS2thin films fabricated by an atmospheric pressure chemical vapor deposition process at ambient temperature. Nanoscale 2014, 6, 12792-12797.

(7) Singh, M.; Haverinen, H. M.; Dhagat, P.; Jabbour, G. E. Inkjet printing-process and its applications. Adv. Mater. 2010, 22, 673-685.

(8) Khan, S.; Doh, Y. H.; Khan, A.; Rahman, A.; Choi, K. H.; Kim, D. S. Direct patterning and electrospray deposition through EHD for fabrication of printed thin film transistors. Curr. Appl. Phys. 2011, 11, S271-S279.

(9) Jaworek, A.; Sobczyk, A.; Krupa, A.; Lackowski, M.; Czech, T. Electrostatic deposition of nanothin films on metal substrate. Bull. Pol. Acad. Sci.: Tech. Sci. 2009, 57, 63-70.

(10) Jayasinghe, S. N.; Townsend-Nicholson, A. Bio-electrosprays: The next generation of electrified jets. Biotechnol. J. 2006, 1, 10181022.

(11) Jaworek, A.; Krupa, A.; Lackowski, M.; Sobczyk, A. T.; Czech, T.; Ramakrishna, S.; Sundarrajan, S.; Pliszka, D. Nanocomposite fabric formation by electrospinning and electrospraying technologies. J. Electrost. 2009, 67, 435-438.

(12) Rha, S.-K.; Chou, T. P.; Cao, G.; Lee, Y.-S.; Lee, W.-J. Characteristics of silicon oxide thin films prepared by sol electrophoretic deposition method using tetraethylorthosilicate as the precursor. Curr. Appl. Phys. 2009, 9, 551-555.

(13) Lenggoro, I. W.; Lee, H. M.; Okuyama, K. Nanoparticle assembly on patterned "plus/minus" surfaces from electrospray of colloidal dispersion. J. Colloid Interface Sci. 2006, 303, 124-130.

(14) Wang, D. Z.; Jayasinghe, S. N.; Edirisinghe, M. J.; Luklinska, Z. B. Coaxial electrohydrodynamic direct writing of nano-suspensions. J. Nanopart. Res. 2007, 9, 825-831.

(15) Chen, C. H.; Kelder, E. M.; Schoonman, J. c2 thin films. Thin Solid Films 1999, 342, 35-41.
(16) Jaworek, A.; Sobczyk, A. T. Electrospraying route to nanotechnology: an overview. J. Electrost. 2008, 66, 197-219.

(17) Ju, J.; Yamagata, Y.; Higuchi, T. Thin-Film Fabrication Method for Organic Light-Emitting Diodes Using Electrospray Deposition. Adv. Mater. 2009, 21, 4343-4347.

(18) Fukuda, T.; Asaki, H.; Asano, T.; Takagi, K.; Honda, Z.; Kamata, N.; Ju, J.; Yamagata, Y. Surface morphology of fluorene thin film fabricated by electrospray deposition technique using two organic solvents: Application for organic light-emitting diodes. Thin Solid Films 2011, 520, 600-605.

(19) Li, W.; Zheng, G.; Xu, L.; Wang, X. Fabrication of micropatterns via near-field electrospray. AIP Adv. 2016, 6, 115002.

(20) Hashimdeen, S. H.; Miodownik, M.; Edirisinghe, M. J. Print head design and control for electrohydrodynamic printing of silk fibroin. Mater. Sci. Eng., C 2013, 33, 3309-3318.

(21) Huang, Z.-M.; Zhang, Y.-Z.; Kotaki, M.; Ramakrishna, S. A review on polymer nanofibers by electrospinning and their applications in nanocomposites. Compos. Sci. Technol. 2003, 63, $2223-2253$

(22) Beachley, V.; Wen, X. Effect of electrospinning parameters on the nanofiber diameter and length. Mater. Sci. Eng., C 2009, 29, 663668

(23) Heseltine, P. L.; Ahmed, J.; Edirisinghe, M. Developments in Pressurized Gyration for the Mass Production of Polymeric Fibers. Macromol. Mater. Eng. 2018, 303, 1800218.

(24) Ferraro, P.; Coppola, S.; Grilli, S.; Paturzo, M.; Vespini, V. Dispensing nano-pico droplets and liquid patterning by pyroelectrodynamic shooting. Nat. Nanotechnol. 2010, 5, 429-435.

(25) Coppola, S.; Vespini, V.; Grilli, S.; Ferraro, P. Self-assembling of multi-jets by pyro-electrohydrodynamic effect for high throughput liquid nanodrops transfer. Lab Chip 2011, 11, 3294-3298.

(26) Coppola, S.; Mecozzi, L.; Vespini, V.; Battista, L.; Grilli, S.; Nenna, G.; Loffredo, F.; Villani, F.; Minarini, C.; Ferraro, P. Nanocomposite polymer carbon-black coating for triggering pyroelectrohydrodynamic inkjet printing. Appl. Phys. Lett. 2015, 106, 261603.

(27) Grimaldi, I. A.; Coppola, S.; Loffredo, F.; Villani, F.; Nenna, G.; Minarini, C.; Vespini, V.; Miccio, L.; Grilli, S.; Ferraro, P. Graded-size microlens array by the pyro-electrohydrodynamic continuous printing method. Appl. Opt. 2013, 52, 7699-7705.

(28) Vespini, V.; Coppola, S.; Todino, M.; Paturzo, M.; Bianco, V.; Grilli, S.; Ferraro, P. Forward electrohydrodynamic inkjet printing of optical microlenses on microfluidic devices. Lab Chip 2016, 16, 326333.

(29) Bianco, V.; Mandracchia, B.; Marchesano, V.; Pagliarulo, V.; Olivieri, F.; Coppola, S.; Paturzo, M.; Ferraro, P. Endowing a plain fluidic chip with micro-optics: a holographic microscope slide. Light: Sci. Appl. 2017, 6, No. e17055.

(30) Jaworek, A.; Sobczyk, A. T. Electrospraying route to nanotechnology: An overview. J. Electrost. 2008, 66, 197-219.

(31) Coppola, S.; Vespini, V.; Nasti, G.; Gennari, O.; Grilli, S.; Ventre, M.; Iannone, M.; Netti, P. A.; Ferraro, P. Tethered PyroElectrohydrodynamic Spinning for Patterning Well-Ordered Structures at Micro- and Nanoscale. Chem. Mater. 2014, 26, 3357-3360.

(32) Coppola, S.; Nasti, G.; Todino, M.; Olivieri, F.; Vespini, V.; Ferraro, P. Direct writing of microfluidic footpaths by pyro-ehd printing. ACS Appl. Mater. Interfaces 2017, 9, 16488-16494.

(33) Grilli, S.; Miccio, L.; Gennari, O.; Coppola, S.; Vespini, V.; Battista, L.; Orlando, P.; Ferraro, P. Active accumulation of very diluted biomolecules by nano-dispensing for easy detection below the femtomolar range. Nat. Commun. 2014, 5, 5314.

(34) Bhat, P. P.; Appathurai, S.; Harris, M. T.; Pasquali, M.; McKinley, G. H.; Basaran, O. A. Formation of beads-on-a-string structures during break-up of viscoelastic filaments. Nat. Phys. 2010, $6,625-631$.

(35) Collins, R. T.; Jones, J. J.; Harris, M. T.; Basaran, O. A. Electrohydrodynamic tip streaming and emission of charged drops from liquid cones. Nat. Phys. 2008, 4, 149-154. 
(36) Park, J.-U.; Lee, S.; Unarunotai, S.; Sun, Y.; Dunham, S.; Song, T.; Ferreira, P. M.; Alleyene, A. G.; Paik, U.; Rogers, J. A. Nanoscale, electrified liquid jets for high-resolution printing of charge. Nano Lett. 2010, 10, 584-591.

(37) Park, I.; Kim, S. B.; Hong, W. S.; Kim, S. S. Classification of electrohydrodynamic spraying modes of water in air at atmospheric pressure. J. Aerosol Sci. 2015, 89, 26-30.

(38) You, X.; Ye, C.; Guo, P. Electric field manipulation for deposition control in near-field electrospinning. J. Manuf. Process. 2017, 30, 431-438.

(39) Vespini, V.; Coppola, S.; Grilli, S.; Paturzo, M.; Ferraro, P. Milking liquid nano-droplets by an IR laser: A new modality for the visualization of electric field lines. Meas. Sci. Technol. 2013, 24, 045203.

(40) Gennari, O.; Battista, L.; Silva, B.; Grilli, S.; Miccio, L.; Vespini, V.; Coppola, S.; Orlando, P.; Aprin, L.; Slangen, P.; Ferraro, P. Investigation on cone jetting regimes of liquid droplets subjected to pyroelectric fields induced by laser blasts. Appl. Phys. Lett. 2015, 106, 054103.

(41) Mahalingam, S.; Edirisinghe, M. Forming of Polymer Nanofibers by a Pressurised Gyration Process. Macromol. Rapid Commun. 2013, 34, 1134-1139.

(42) He, X.-X.; Zheng, J.; Yu, G.-F.; You, M.-H.; Yu, M.; Ning, X.; Long, Y.-Z. Near-field electrospinning: progress and applications. J. Phys. Chem. C 2017, 121, 8663-8678.

(43) Nithyanandan, A.; Mahalingam, S.; Huang, J.; Rehman, S.; Draper, E.; Edirisinghe, M. Template-assisted electrohydrodynamic atomization of polycaprolactone for orthopedic patterning applications. Mater. Sci. Eng., C 2013, 33, 4608-4615.

(44) Wang, D. Z.; Jayasinghe, S. N.; Edirisinghe, M. J. Instrument for electrohydrodynamic print-patterning three-dimensional complex structures. Rev. Sci. Instrum. 2005, 76, 075105.

(45) Zhu, T.; Li, C.; Yang, W.; et al. Electrospray Dense Suspensions of TiO2 Nanoparticles for Dye Sensitized Solar Cells. Aerosol. Sci. Technol. 2013, 47, 1302-1309.

(46) Gennari, O.; Marchesano, V.; Rega, R.; Mecozzi, L.; Nazzaro, F.; Fratianni, F.; Coppola, R.; Masucci, L.; Mazzon, E.; Bramanti, A.; Ferraro, P.; Grilli, S. Pyroelectric Effect Enables Simple and Rapid Evaluation of Biofilm Formation. ACS Appl. Mater. Interfaces 2018, $10,15467-15476$.

(47) Xu, Z. Gyration Spun Polymeric Fibres for Antibacterial Applications, Ph.D. Dissertation, University College London, London, 2017.

(48) Matharu, R. K.; Charani, Z.; Ciric, L.; Illangakoon, U. E.; Edirisinghe, M. Antimicrobial activity of tellurium-loaded polymeric fiber meshes. J. Appl. Polym. Sci. 2018, 135, 46368.

(49) Nguyen, V. H.; Kim, B.-K.; Jo, Y.-L.; Shim, J.-J. Preparation and Antibacterial Activity of Silver Nanoparticles-Decorated Graphene Composites. J. Supercrit. Fluids 2012, 72, 28-35.

(50) Xu, Z.; Mahalingam, S.; Rohn, J. L.; Ren, G.; Edirisinghe, M. Physio-chemical and antibacterial characteristics of pressure spun nylon nanofibres embedded with functional silver nanoparticles. Mater. Sci. Eng., C 2015, 56, 195-204.

(51) de Faria, A. F.; Martinez, D. S. T.; Meira, S. M. M.; de Moraes, A. C. M.; Brandelli, A.; Filho, A. G. S.; Alves, O. L. Anti-Adhesion and Antibacterial Activity of Silver Nanoparticles Supported on Graphene Oxide Sheets. Colloids Surf., B 2014, 113, 115-124.

(52) Markowska, K.; Grudniak, A. M.; Wolska, K. I. Silver Nanoparticles as an Alternative Strategy against Bacterial Biofilms. Acta Biochim. Polym. 2013, 60, 523-530.

(53) Shao, W.; Liu, X.; Min, H.; Dong, G.; Feng, Q.; Zuo, S. Preparation, Characterization, and Antibacterial Activity of Silver Nanoparticle-Decorated Graphene Oxide Nanocomposite. ACS Appl. Mater. Interfaces 2015, 7, 6966-6973. 